Check for updates

Cite this: Phys. Chem. Chem. Phys., 2019, 21, 4888

Received 10th September 2018 Accepted 12th November 2018

DOI: $10.1039 / c 8 c p 05720 f$

rsc.li/pccp

\title{
A genomic characterisation of monometallic nanoparticles
}

\author{
Kevin Rossi, (D) Gian Giacomo Asara and Francesca Baletto (D) *
}

\begin{abstract}
Because size and shape can affect the chemo-physical properties of nanoparticles, we extend the use of geometrical descriptors to sequence a genome of monometallic nanoparticles. Selecting the generalised coordination number as a descriptor, the derived geometrical genome distinguishes, catalogues, and counts the variety of adsorption sites available on each isomer with a diameter up to $10 \mathrm{~nm}$, therefore it depends on the nanoparticle size and shape. This procedure allows us to elucidate the effects of morphological diversity within a sample and those of thermally activated structural rearrangements among isomers on nanocatalyst activity. By screening the geometrical genome of archetypal shapes, we forecast Pt stellated twinned nanoparticles, elongated along their five-fold axis and with their shortest diameter of $\sim 2 \mathrm{~nm}$, as optimal candidates for the electro-reduction of molecular oxygen at room temperature, in agreement with available experimental data.
\end{abstract}

\section{Introduction}

Chemical reactions often consist of many intermediate steps and happen on so-called active sites, which might be only a small fraction of the total surface of a catalyst. ${ }^{1-4}$ As the mechanistic study of each intermediate step of a reaction per each site is highly demanding with standard computational tools, ${ }^{5}$ a fast screening of materials with ad hoc catalytic properties proceeds using descriptors, which establish a correlation between the catalytic activity and intrinsic properties of the sites where the reaction occurs. ${ }^{6,7}$ Following Sabatier's principle, the adsorption energy is a meaningful descriptor to screen materials as catalysts. At the same time, reaction free energies have been shown to provide an upper limit of electrochemical activity. ${ }^{7}$ In recent years, many efforts have been pursued in finding measurable quantities that give useful structure-activity relationships. These physical entities can be both geometrical - e.g. coordination number, electronic - e.g. d-band centre-, or a mixture of them - e.g. orbital-wise coordination number. ${ }^{8-10}$ The final objective is the use of those quantities to translate our theoretical and atomistic understanding of matter into attractive rational rules to design catalysts overcoming serendipity - or at least reducing it - in finding optimal catalysts for a target reaction. ${ }^{11,12}$

The need to map adsorption, reaction, and activation free energies is more drastic at the nanoscale, where the lack of any translation symmetry leads to the coexistence of many isomers interconnected by thermally activated structural rearrangement

Physics Department, King's College London, Strand, WC2R 2LS, UK.

E-mail: francesca.baletto@kcl.ac.uk mechanisms, as recently confirmed by microscopy and spectroscopy in situ studies of metallic nanoparticles (mNPs). ${ }^{13,14}$ Notwithstanding technical challenges in controlling matter at the nanoscale, there is potential to tune the catalytic performance of mNPs by playing with their shape, size and chemical composition and also exploiting the fact that they are structurally dynamic objects. ${ }^{15,16}$ In this view, the use of mNPs to catalyse more efficiently and selectively chemical reactions has the potential to trigger a proper revolution in the green economy. Two factors should be simultaneously taken into account: (i) the variety of adsorption sites per each isomer, ${ }^{17}$ which influences its activity and selectivity, and (ii) the coexistence of different geometries, eventually related to their formation process. ${ }^{18}$ Numerical guidelines enable researchers to pursue the synthesis of samples with a well defined and optimal architecture (size, shape, chemical composition and ordering) avoiding a costly and ineffective trial-and-error procedure. If, at first glance, this appears as an optimisation problem, the main challenge resides in the amount of calculations needed towards this end, which is out-of-reach. This requires the development of alternative numerical strategies. Effective tailoring schemes should enable the identification of mNPs presenting an optimal kind and occurrence of active sites, while accounting for the intrinsic morphological heterogeneity of a sample and the variety of adsorption sites of each isomer.

Here, we provide a high-throughput geometrical analysis of closed-shell Pt nanoarchitectures up to $10 \mathrm{~nm}$ in diameter, whose adsorption sites are classified according to their generalized coordination number (GCN) for both atop and bridge adsorptions. Our numerical strategy promises to bridge the gap between $a b$ initio predictions and a fast and reliable screening 
of nanoarchitectures for a target application, while also accounting for the effect of thermally activated structural rearrangements, diffusion, and morphological heterogeneity in a sample. The application of our approach to probe the catalytic properties of low-symmetry nanoarchitectures observed at nonmagic sizes or following thermally driven surface roughening is indeed also discussed in the manuscript.

As a paradigmatic key study, we focus on the oxygen reduction reaction (ORR). The latter is the sluggish electrochemical process occurring at the cathode and it is regarded as one of the bottlenecks for a wide range of proton exchange membrane fuel cells. It has been shown that Pt nanoparticles can enhance this reaction, ${ }^{19,20}$ but we are still far from improving its efficiency by $\sim 30 \%$, the target which would make such fuel cells economically competitive against today engines. ${ }^{21}$ After the chemisorption of molecular oxygen, the ORR involves many intermediates - e.g. $\mathrm{O}_{2}$ splitting, $\mathrm{OOH}$ or $\mathrm{OH}$ formation, which lead to the formation of water. State-of-the-art $a b$ initio calculations demonstrated that the generalised coordination number is a powerful descriptor to predict the adsorption energy of oxygenated molecules on gas phase $\mathrm{Pt}^{22-25}$ and oxide supported $\mathrm{PtNi}^{26,27}$ nanoparticles: there exists a linear relationship between the binding energy of ORR intermediates at the adsorption site and the corresponding GCN value, i.e. the bonding weakens when increasing the coordination of the active site..$^{2,23,26,28}$ Following the screening of structures commonly observed in experiments, we predict Pt stellated twinned decahedra, elongated along their five-fold axes, as the best morphology for ORR, between 2 and $9 \mathrm{~nm}$. Our predictions are in very good agreement with the recent experimental results that pointed out the enhanced catalytic activity of complex twinned nanoarchitectures when compared to standard $\mathrm{Pt} / \mathrm{C}$ catalysts. ${ }^{29-31}$

\section{Methodology}

In parallel to genetics, we associate to each nanoparticle non equivalent adsorption site (NEAS) a unique tag, e.g. the value of some geometrical descriptor (GD) that uniquely discriminates it from the other sites. Each NEAS then corresponds to a gene in the nanoparticle. In turn, the ensemble of all the kinds of NEAS a nanoparticle shows, determines its fingerprint. Conversely, the nanoparticle genome collects the full catalogue of the adsorption sites it presents, together with their occurrence. The catalytic properties of a nanoparticle can be thus predicted from its genome when the NEASs are distinguished by a proper descriptor, which uniquely and accurately maps the catalytic and geometric properties of any adsorption site.

The prediction of the catalytic properties of a nanoparticle by means of a geometrical analysis finds an accurate result only upon the suitable choice of geometrical descriptor. Trivially, sites with different catalytic properties should be uniquely mapped to different GD values. The necessary condition is that the descriptor correctly classifies all the different nanoparticle adsorption sites, as a function of its size and shape. Indeed, gathering information from the local properties of each active

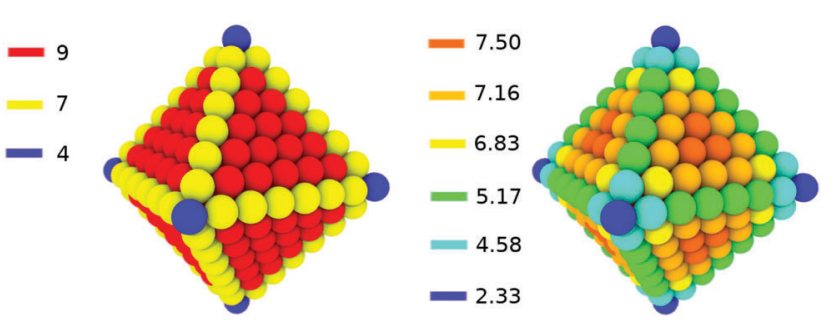

Fig. 1 Colour coded mapping of an atop adsorption site in a regular octahedron, distinguished according to its nominal (left) and generalised (right) coordination. Each NEAS GD value is reported the left of each structure.

site will in turn allow rationalisation of the global catalytic activity of the mNPs as a function of their size and shape. To exemplify the importance of an accurate GD choice, Fig. 1 presents the classification of the atop adsorption sites - when the ad-molecule interacts with one metallic atom, sitting "atop" of it - of an octahedron of 344 atoms according to their nominal and generalized coordination.

For the case of the electrochemical reduction of oxygen and carbon dioxide, which involves the atop adsorption of $\mathrm{OH}, \mathrm{O}$, and $\mathrm{CO}$, the generalized coordination number, GCN, has been proven to be a robust descriptor both on monometallic and bimetallic nanoparticles, supported and in the gas-phase, although still in need of confirmation for ternary alloys. ${ }^{22-27,32,33}$

For an atop site $i, \mathrm{GCN}_{i}$ is calculated as the sum of the coordination number $\mathrm{CN}_{j}$ of each $j$ nearest-neighbour of the underneath metallic atom, normalized with respect to the bulk coordination $\mathrm{CN}_{\max }, 12$ in the case of face centered cubic lattices:

$$
\mathrm{GCN}_{i}=\sum_{j} \frac{\mathrm{CN}_{j}}{\mathrm{CN}_{\max }}
$$

The coordination number of an atom $j, \mathrm{CN}_{j}$, is the number of metallic atoms lying within a sphere of radius $d_{0}$ and centred around it, calculated in an analytic way:

$$
\mathrm{CN}_{j}=\sum_{i \neq j} f\left(r_{i j}\right), \quad f\left(r_{i j}\right)=\left\{\begin{array}{lll}
1 & \text { if } & r_{i j} \leq d_{0}, \\
0 & \text { if } & r_{i j}>d_{0},
\end{array} .\right.
$$

The cut-off distance $d_{0}$ is set at 1.2 times the bulk nearest neighbour distance. This is a good choice even during finite temperature simulations, as it is robust against nearestneighbour bond elongations and contraction of the secondneighbour distances due to the ionic motion. ${ }^{34}$

As clearly seen in Fig. 1, the details encoded in the GCN-map allow us to distinguish sites on the vertexes, edges, and facets close to edges and vertexes from their surface-like counterparts. Moreover, the GCN formalism can be extended to map bridge as well as hollow sites, where the $\mathrm{CN}_{\max }$ is adjusted to match the number of neighbours of pairs or triplets of atoms involved in the adsorption of a molecule. In the bulk, pairs, triplets, and quartets of atoms have a maximum coordination of $\mathrm{CN}_{\max }=$ 18,22 , and 26, respectively. ${ }^{22}$ Atop, bridge, and hollow sites are the modes by which a simple ad-molecule interacts with a clean, metallic surface, as visually depicted in Fig. 2. For molecules 

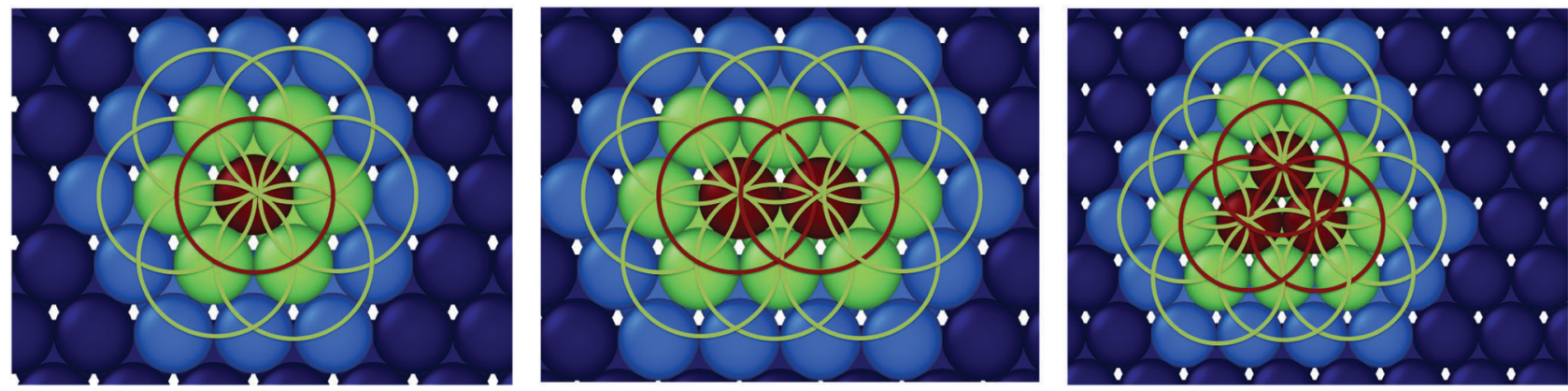

Fig. 2 Visual representation of the generalized coordination estimate for atop (atop GCN), bridge (bridge GCN) and hollow (hollow GCN) sites on a (111) surface. Atoms underneath the site are coloured in red, their first nearest neighbours are shown in light-green, and the nearest neighbours of the latter are shown in light-blue, while all the other atoms are dark blue. Nearest neighbour shells are highlighted by a light-green circle.

adsorbed "atop", the site involves one metallic atom, for a bridge site, the anchor of the ad-molecule lies between them and, similarly, a hollow site comprises three atoms on a (111) surface or four on a (100) surface. We note that on a nanoparticle, adsorption sites show different GCN values depending on their vicinity to an edge, corner, vertex, as well as the dimension and shape of the facet. We stress that thermally activated atomic vibrations even at high temperatures do not strongly alter the definition of a cut-off nearest neighbour distance, which is robust even in the liquid phase. The calculus of the GCN over a wide range of temperatures is robust and the estimated GCN-genome provides information on the appearance of defects, due to surface peeling and surface reconstructions taking place at high- $T$, as they might be characterised by a spreading of GCN blueprints, e.g. in the presence of adatoms and defects, as discussed later.

It is worth noting that the GCN mapping might fail in encoding a good correlation between the geometrical characterisation of sites and adsorption energies of a longer molecule on small clusters, e.g. ethanol, as the relative position and orientation of the alkyl group with respect to the nanoparticle could induce non negligible structural distortions within the cluster that significantly affect the binding energy of the admolecule and the nanocatalyst. This might be a size effect ${ }^{35}$ or a chemical effect due to the Pt-skin over another metallic core. ${ }^{36}$

Regarding the rational design of multi-metallic nanoalloys, a prominent route towards the synthesis of nanocatalysts with bespoken catalytic properties and high stability, ${ }^{37,38}$ let us here remark that the proposed genomic framework is transferable to the application of any suitable geometrical descriptor in the sequencing of adsorption sites on a nanocatalyst. We do not expect qualitative changes if another descriptor is employed when it satisfies the stated requirements of being sensitive to size, shape and of being able to classify non-equivalent adsorption sites. For example, the orbital-wise coordination number, ${ }^{10}$ strain modified GCN,${ }^{39}$ effective coordination,${ }^{40}$ and coordination calculated via exclusion methods ${ }^{41}$ are among the most notable descriptors for predicting the catalytic properties of multi-metallic systems, where the definition of a cut-off distance to estimate the coordination number of surface atoms may be hindered by the overlap of the first and second nearest neighbour peaks due to the mismatch between chemical species. Finally, the strain is often used to predict the activity of bi- or ternary nanoalloys. This quantity is defined as the sum of the ratio between the lattice parameter in the nanoparticle and its reference bulk value. Although it is possible that different shapes lead to a different strain-map, it might be challenging to distinguish adsorption modes and to classify all the various adsorption sites. The use of nanoparticle distortion, i.e. radial breathing, might be included, as in ref. 36 . Nonetheless, ongoing works on the use of the genomic framework on nanoalloys are out of the aim of this work and will be treated in dedicated future projects.

\section{Results}

With a focus on platinum nanoparticles for ORR, we sequence the NEAS kind and occurrence in different structural motifs commonly observed in experiments, as such the cube $(\mathrm{Cb})$, the octahedron $(\mathrm{Oh})$, its regular truncation (rTo), the cubocathedron (Co), the icosahedron (Ih), and cuts of a pentagonal bi-pyramid, in the literature known as the Ino-Decahedra (IDh), and the Marks-Decahedra (MDh). Far from exhaustive of all available structural motifs, we believe that the ensemble of morphologies under scrutiny provides a sufficient structural variety to test the effectiveness of our approach: the study of the nanoparticle geometrical genome to predict its catalytic activity. Furthermore, starting from the ideal single motif case, we also investigate the effect of surface peeling, surface roughening and structural changes, as well as how the geometrical genome description can be extended to predict the morphological behaviour of diverse samples.

\subsection{Perfect closed-shell nanoparticles as nanocatalysts}

Cubic, octahedral, icosahedral, Ino and Marks-decahedral structures alongside the size evolution of their fingerprint are depicted in Fig. 3.

We observe a general pattern in the size dependent evolution of the list of NEASs (as distinguished by their GCN) characteristic of each structure. Independently of the shape and adsorption mode, we identify three size-regions according 

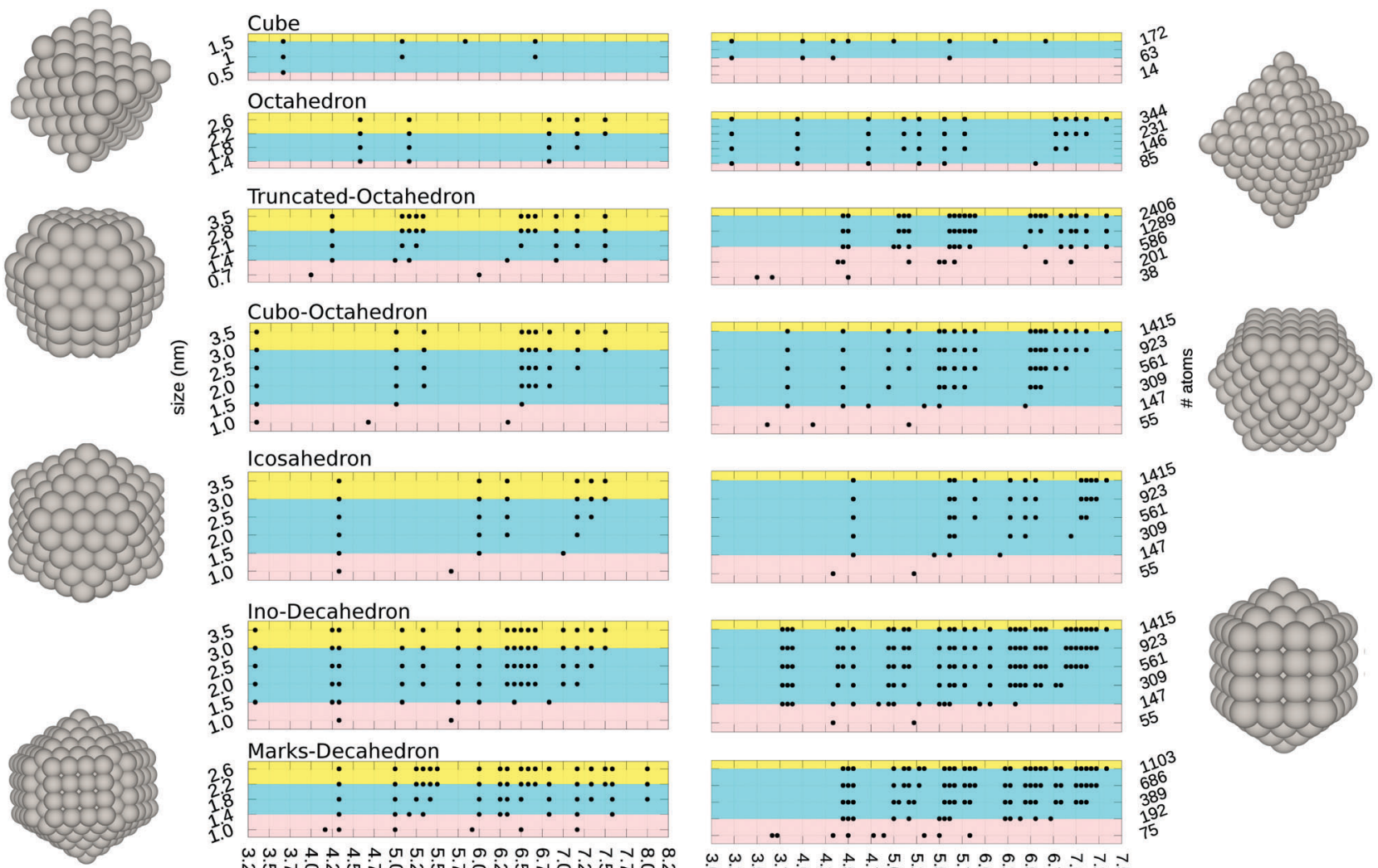
जुण जै

GCN (atop)
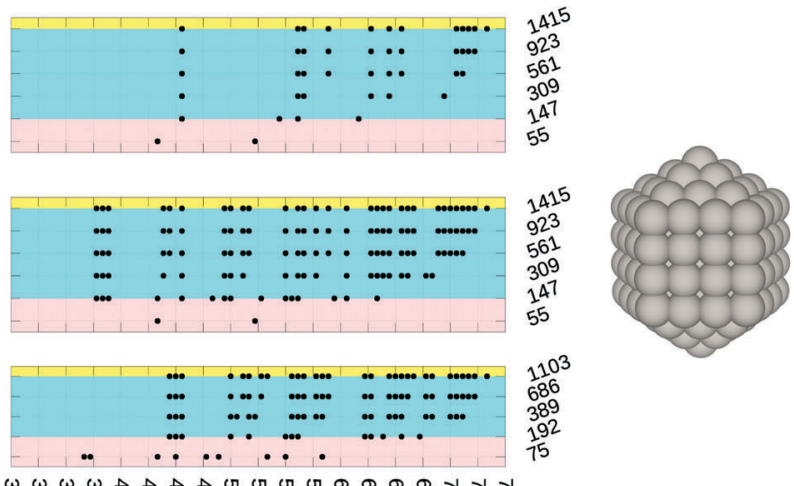

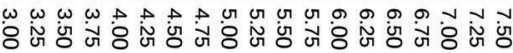

GCN (bridge)

Fig. 3 Nanoparticle GCN fingerprint size evolution in the seven structures under investigation (a snapshot of each is reported alongside the graph), for atop (left) and bridge (right) adsorptions. The three size regimes, where the fingerprint is fixed (yellow), incomplete with respect to larger sizes (cyan), and presenting NEASs of unique small sizes (pink), are colour coded.

to the diameter of the cluster, $d_{\mathrm{cl}}$, highlighted in the plot in different colours:

- $d_{\mathrm{cl}}>3.5 \mathrm{~nm}$, all the considered shapes show a GCN fingerprint, which constitutes a distinctive geometrical attribute of that morphology.

- $1.5 \mathrm{~nm}<d_{\mathrm{cl}}<3.5 \mathrm{~nm}$, the GCN fingerprint is similar to the ones of larger size nanoparticles, but it does not present all the NEASs therein because the facets are not large enough to accommodate all the different active sites, e.g. GCN $>7$ is not shown yet.

- $d_{\mathrm{cl}}<1.5 \mathrm{~nm}$, only a few NEASs available, the GCN fingerprint is strongly different from the ones of larger sizes, with NEASs presenting GCNs uniquely characteristic of nanoparticles of a given nuclearity.

The three size-regimes can be related to the cluster size at which the electronic charge distribution at an active site is likely to be similar to its infinite/periodic surface counterpart, if existing, and to predict the properties of sites in systems prohibitively expensive to be studied at the ab initio level from the ones in smaller clusters. Following the GCN genome sequencing, the adsorption of atomic $\mathrm{O}$ onto (111)- and (211)-like NEASs in a Pt nanoparticle, e.g. a cuboctahedral morphology and a nuclearity of at least 309 atoms, will resemble their extended surface counterparts. This prediction is in good agreement with electronic structure calculations reported by Nørskov and coworkers. ${ }^{42}$ In the subnanometer size regime, the appearance of peculiar properties, due to significant changes in the cluster electronic structure, is expected and depends on each atom. This opens a wide range of opportunity, the investigation of which is out of the scope of the present research paper.

Size and shape interplay drives the relative occurrence of a NEAS. Vertex and close-to vertex sites are the first to appear, but their number is fixed. They are followed by the edge NEAS and close-to-edge NEAS, which scale linearly with size. Finally, surface-like NEASs appear. Their occurrence presents a quadratic dependence with respect to cluster nuclearity. We also note that nanoparticles presenting facets with a larger (smaller) area to perimeter present a complete GCN fingerprint earlier (later). To exemplify this discussion, we report the NEAS size-occurrence scaling laws for the paradigmatic case of an octahedron, for both atop and bridge site, in Fig. 4. In particular, here, we show how to predict NEAS occurrence as a function of the number of atoms along the edge.

A non-trivial size dependence of the NEAS relative occurrence thus arises from the aforementioned geometrical consideration. We report the detailed discussion of the size-dependent relative abundance of NEASs in Oh nanoparticles in Fig. 5. At $d_{\mathrm{cl}} \sim 2.6 \mathrm{~nm}$, 344 atoms, we observe an almost homogeneous relative occurrence 
Octahedron

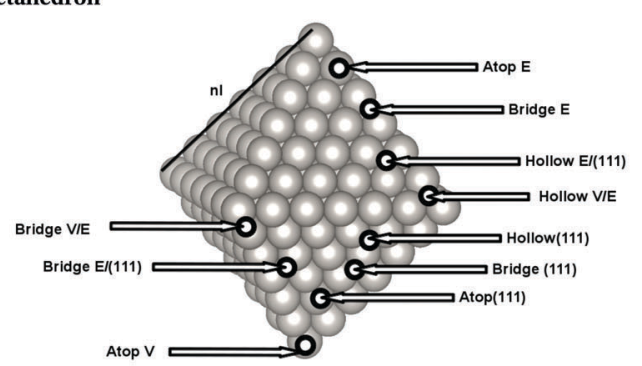

Total atoms on (111) facet $=8 \times[((\mathrm{nl}-3)(\mathrm{nl}-2)) / 2]$ Total edge atoms $=12 \times(\mathrm{nl}-2)$

\begin{tabular}{|c|c|}
\hline Adsorption Site & N\# Adsorption Sites (i.e. O) \\
\hline Atop V & 6 \\
\hline Atop E & $12(\mathrm{nl}-2)$ \\
\hline Atop (111) & $4(\mathrm{nl}-3)(\mathrm{nl}-2)$ \\
\hline Bridge V/E & 24 \\
\hline Bridge E & $12(\mathrm{nl}-3)$ \\
\hline Bridge E/(111) & $48(\mathrm{nl}-3)$ \\
\hline Bridge (111) & $8[(3 / 2)(\mathrm{nl}-4)(\mathrm{nl}-3)]$ \\
\hline Hollow V/E & 24 \\
\hline Hollow E/(111) & $8(3(\mathrm{nl}-3)+3(\mathrm{nl}-4))$ \\
\hline Hollow(111) & $4((\mathrm{nl}-4)(\mathrm{nl}-3)+(\mathrm{nl}-5)(\mathrm{nl}-4))$ \\
\hline
\end{tabular}

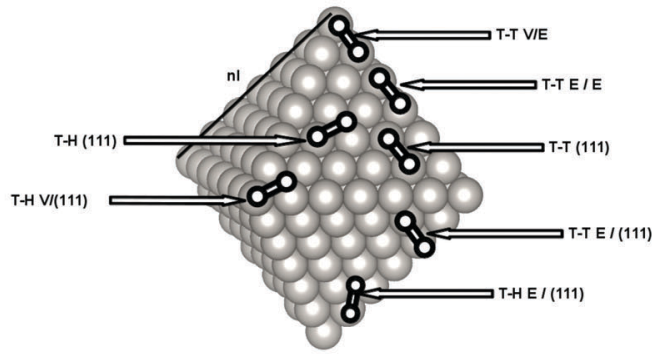

\begin{tabular}{|c|c|}
\hline Adsorption Site & N\# Adsorption Sites (i.e. $\left.\mathrm{O}_{2}\right)$ \\
\hline T-T V/E & 24 \\
\hline T-T E/E & $6(\mathrm{nl}-2)$ \\
\hline T-T E $/(111)$ & $8[(\mathrm{nl}-3)+(\mathrm{nl}-4)+(\mathrm{nl}-5)]$ \\
\hline T-T $(111)$ & $2(\mathrm{nl}-3)(\mathrm{nl}-2)$ \\
\hline T-H $(111)$ & $24[\mathrm{Integer}(1 / 3((\mathrm{nl}-3)(\mathrm{nl}-2) / 2)]$ \\
\hline T-H V $/(111)$ & 24 \\
\hline T-H E $/(111)$ & $24(\mathrm{nl}-3)$ \\
\hline & \\
\hline & \\
& \\
\hline
\end{tabular}

Fig. 4 A visual representation of the non equivalent adsorption sites for atop (left) and bridge (right) adsorptions in an Oh. For each case, we report polynomial expressions that allow us to estimate each NEAS occurrence as a function of the length (in terms of the number of atoms, $n_{l}$ ) of the Oh edge.
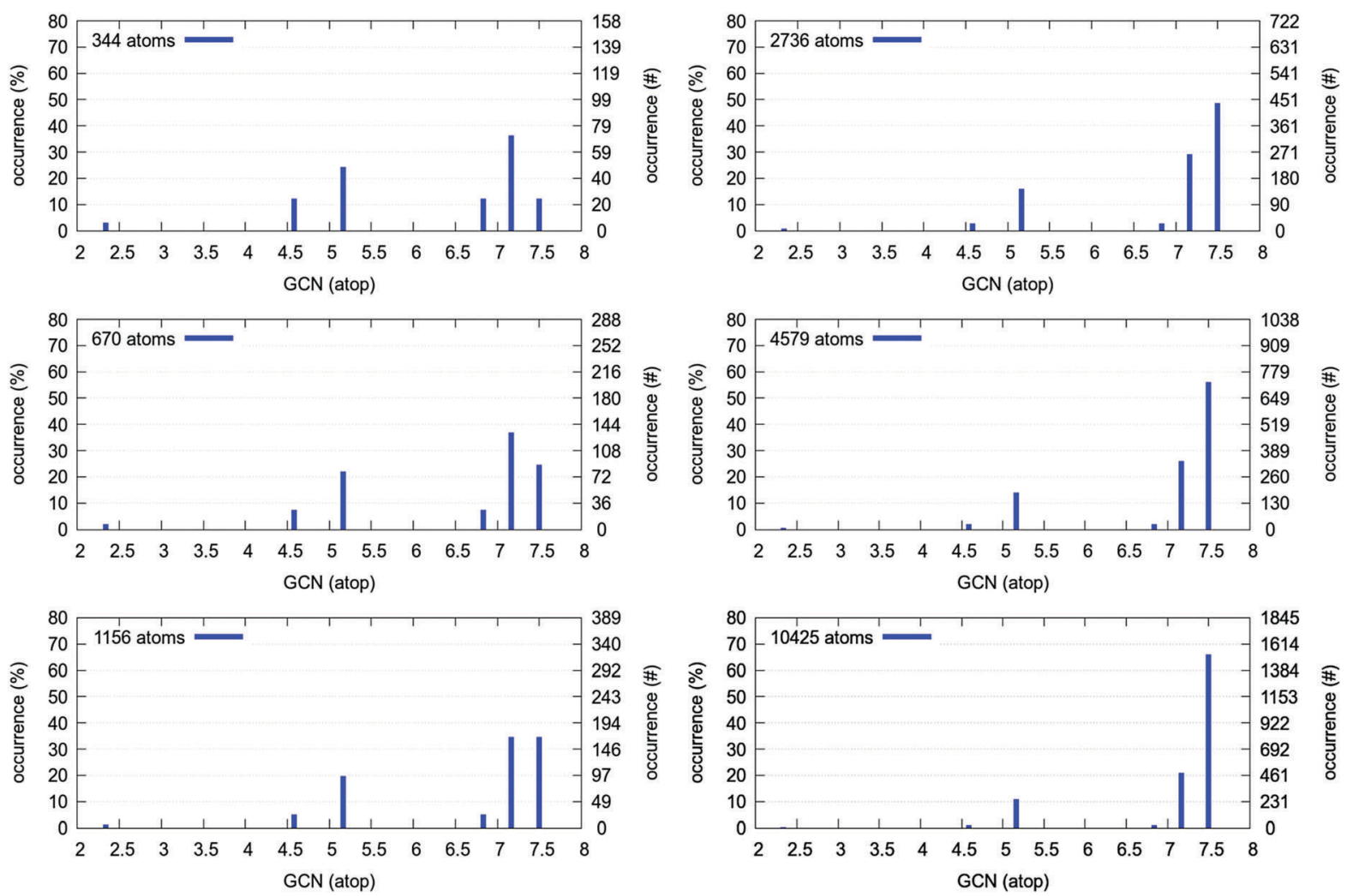

Fig. 5 Atop GCN genome for Oh at different sizes ( $2.7 \mathrm{~nm}, 3.5 \mathrm{~nm}, 4.3 \mathrm{~nm}, 5.8 \mathrm{~nm}, 7 \mathrm{~nm}, 9.5 \mathrm{~nm}$ ), where the relative (nominal occurrence divided by the total number of sites) and nominal occurrence are provided.

of each NEAS, with active sites with a GCN close to 7 slightly more frequent than others. At $d_{\mathrm{cl}}=4.3 \mathrm{~nm}$, corresponding to a cluster nuclearity of 1156 atoms, (111) surface sites $(\mathrm{GCN}=7.5)$ and NEASs with GCN $=7.16$ present a comparable occurrence. The favourable scaling of (111)-like NEASs makes them more frequent at larger sizes. Indeed, at $d_{\mathrm{cl}}=7 \mathrm{~nm}$ (4579 atoms), surface site 
relative occurrence is almost twice the one of the second most abundant one. By the same token, for a cluster size of 10425 atoms, $d_{\mathrm{cl}}=9.5 \mathrm{~nm},(111)$ sites account for almost $70 \%$ of the total.

Features in the GCN fingerprint can be rationalized in terms of the cluster morphology and size. When delimited by a variety of facets, nanoparticles show a diversification of their NEASs with the MDh having the largest number due to the presence of both (111), (100), and re-entrances. $\mathrm{Cu}, \mathrm{Oh}$ and $\mathrm{Ih}$ present a smaller number of different NEASs being terminated only by either (100) or (111) facets. Co, IDh, and To display a larger number of NEASs because they are limited by both (100) and (111) facets. Motifs that share a given local environment also present NEASs with the same GCN value. For example, the active sites with $\mathrm{GCN}$ (atop) $=7.5$, found in all the shapes here considered apart from $\mathrm{Cu}$, lie on (111) facets. Similarly, active sites with $\mathrm{GCN}$ (atop) $=6.66$, corresponding to the sites on (100) surfaces, are identified in all apart Ih and Oh. Only concave structures present NEASs with GCN (atop) $>7.5$. The result of this geometric consideration is captured by the Circos-plot shown in Fig. 6, which reports on the left hand side the non equivalent active sites and in the right section, the seven different geometries (of size $\sim 1000$ atoms) under scrutiny, and a band connecting them if the former belongs to the latter.

\subsection{Beyond the ideal closed-shell single motif picture}

To provide design guidelines beyond the static and ideal motif pictures, closer to what is observed under experimental conditions, we propose two gedanken experiments: the first is devoted to revealing the effect of geometrical polydispersity of a size-selected sample, and the second aims to elucidate the role of vibrations, surface diffusion and shape fluctuations at finite temperatures. The latter suggests the need for developing

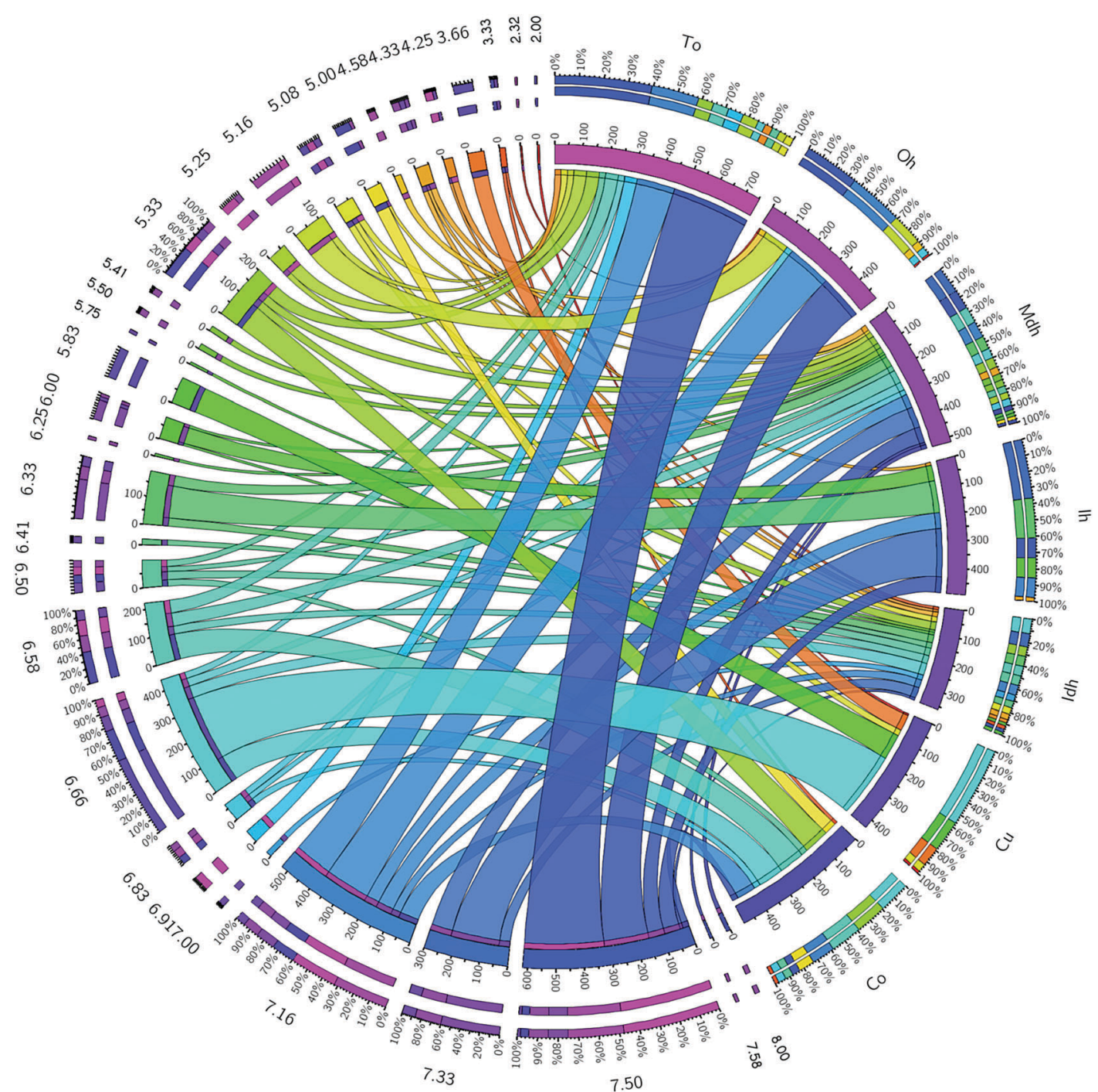

Fig. 6 Circos-plot to highlight the relationships between NEASs, labelled according to their GCN value, and the isomers at $\sim 3.5 \mathrm{~nm}$. Bands of different colours connect NEAS (left portion of the circle) and mNP morphology (right portion of the circle). For all sites, we report their relative occurrence in \% (outer circle) and occurrence (inner circle). Band colour coding facilitates the identification of the NEAS relative occurrence in a given shape. 
models that include mobility of the surface atoms and the reconstruction of adsorption sites. The former calls for a comprehensive investigation of the effect of morphological diversity in ensembles of clusters, and how it affects their catalytic properties.

3.2.1 From perfect to defected: surface roughening analysis. To move forward the analysis of structures presenting a closedshell geometry and before analysing the effect of structural heterogeneity in a sample and rearrangements in a nanoparticle, let us here discuss the analysis of the genomes observed in 20 structures obtained by peeling atoms from the surface of a cubo-octahedron of 309 atoms, down to 289 atoms. The choice of this case study allows us to show how the GCN genome sequencing is able to address the challenges posed by the presence of many NEASs peculiar of low-symmetry systems. Further, it finds an immediate comparison to experimental results where similar low-symmetry, surface roughened structures have been observed. ${ }^{43}$ Low-symmetry defected structures are here constructed by iteratively removing atoms from the surface of the nanoparticle down to the desired nuclearity, obtained through a stochastic algorithm. The probability that an atom is peeled off the cluster surface is determined from its generalized coordination, with low coordinated atoms more likely to be removed from the nanoparticle surface. This choice finds a match in the experimental observation of low coordinated atom dissolution from the nanoparticle, especially for the case of strongly acidic environments.

Fig. 7 shows the genome of 20 defected architectures obtained by peeling a perfect cuboctahedron of 309 atoms. While most, if not all, of the NEASs observed in the closed-shell mother structure appear in the defected structures too, the latter present a much rougher GCN-genome with the appearance of several NEASs unique of low-symmetry shapes. From the comparison of the genomes, we note that the peeling of low-coordinated atoms from a closed-shell geometry allows

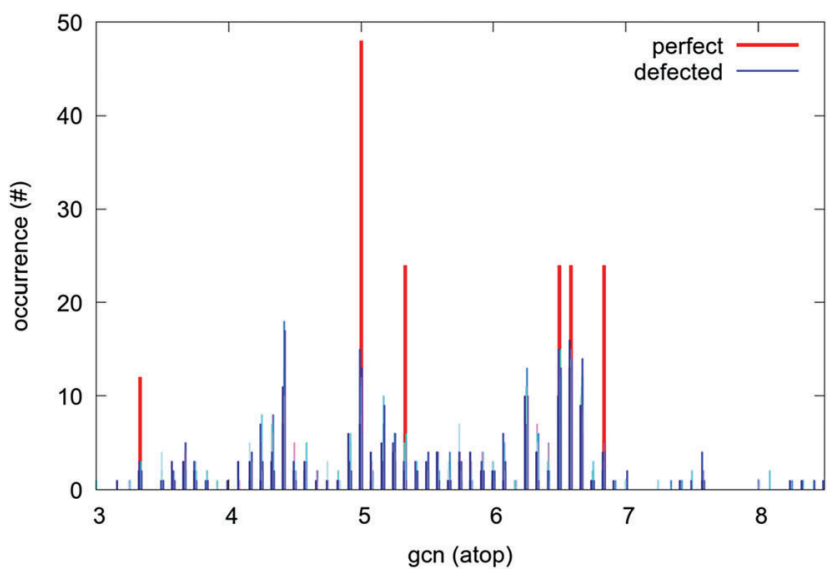

Fig. 7 A visual representation of the atop GCN genomes of a perfect closed-shell cuboctahedron of 309 atoms (red) and its 10 related non closed-shell nanoparticles (shades of blue) obtained by removing the 20 lowest coordinated atoms from it. Atom removal results in a reduction of the atomic coordination of some surface atoms and in the exposure of highly coordinated subsurface atoms. previously subsurface sites to become available as molecular adsorption sites. These 'new' sites, reminiscent of vacancies, are characterised by a high GCN-value and might turn out to be active for ORR, in agreement with previous prediction. ${ }^{23}$

3.2.2 From one to many: morphological sample analysis. To elucidate how the GCN genome depends on the morphological heterogeneity in a sample, we compare three populations of Pt-nanoparticles with $575 \pm 20$ atoms, as shown in the top panel of Fig. 8. Beyond perfectly closed-shell structures, low-symmetry nanoparticles are also commonly observed alongside their high-symmetry counterparts in experiments. From energetic considerations based on the standard and modified Wulff construction, ${ }^{44}$ the MDh of 585 atoms is expected to be energetically favourable. On the other hand, geometrical intuition makes Ih and To, of 561 and 586 atoms, respectively, possible alternatives, which turn out to be energetically competitive with the MDh. We note that at these sizes, Ih still has a negligible number of surface-like sites; it already has $20-25 \%$ of highly coordinated sites, and MDh allows for the adsorption of molecules in its re-entrances.

For this reason, three example populations are considered. Population Fig. 8(i), in red, is described by a unimodal sizedistribution peaked around 585 atoms and with a standard deviation of 5 atoms. It is composed of an $80 \%$ equal split between $\mathrm{MDh}_{585}$ and $\mathrm{To}_{586}$, respectively, while the remaining $20 \%$ is low energy isomers with 580 and 590 atoms. These are identified by means of a basin-hopping search. ${ }^{45}$ For population Fig. 8(ii), in orange, we allow a standard deviation of 15 atoms with $60 \%$ of $\mathrm{MDh}_{585}$ and $\mathrm{To}_{586}$, another $30 \%$ made of the putative global minima structures at 580 and 590 atoms, respectively, and the last $10 \%$ of energetically favourable nanoparticle shapes of 595 and 575 atoms. Population Fig. 8(iii), in blue, displays a bimodal distribution of closed-shell geometries centred at 561, where Ih should be energetically favourable, and at 585 and 586 atoms, where we find respectively a MDh and a rTo. The relative abundance of each structure is $50 \%$ for Ih and 25\% each for MDh and To.

When analysing the GCN genome of each population, we note that samples of closed-shell structures, as the blue population, are characterised by fewer NEASs, with overall higher GCN values. Introducing defected shapes, with a variety of re-entrances, steps, and islands, the GCN genome shows a broadened distribution, with tails towards both smaller GCN values as well as higher ones $(>7.5)$ due to the presence of concave adsorption sites. By comparing the red and orange populations, we note that even a small change in the cluster size and shape distribution may significantly affect the population of the GCN genome, and hence a non-negligible difference in their catalytic performance is expected. When the size distribution and the morphological heterogeneity of the ensemble are broadened, the GCN values centred around 5 decrease from $25 \%$ to $20 \%$, and the percentage between those between 7 and 8 falls from $50 \%$ to $30 \%$. We can extrapolate the qualitative catalytic behaviour of a sample by looking at its ensemble GCN genome: a morphologically diverse catalyst is likely to show a lower selectivity, with many side-reactions taking place. An increase in the sample 


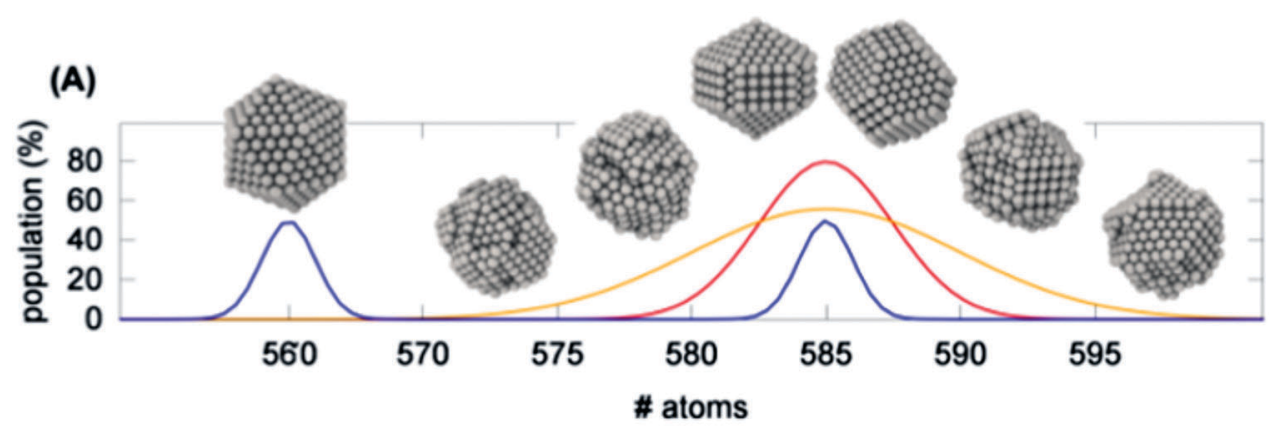

(B)
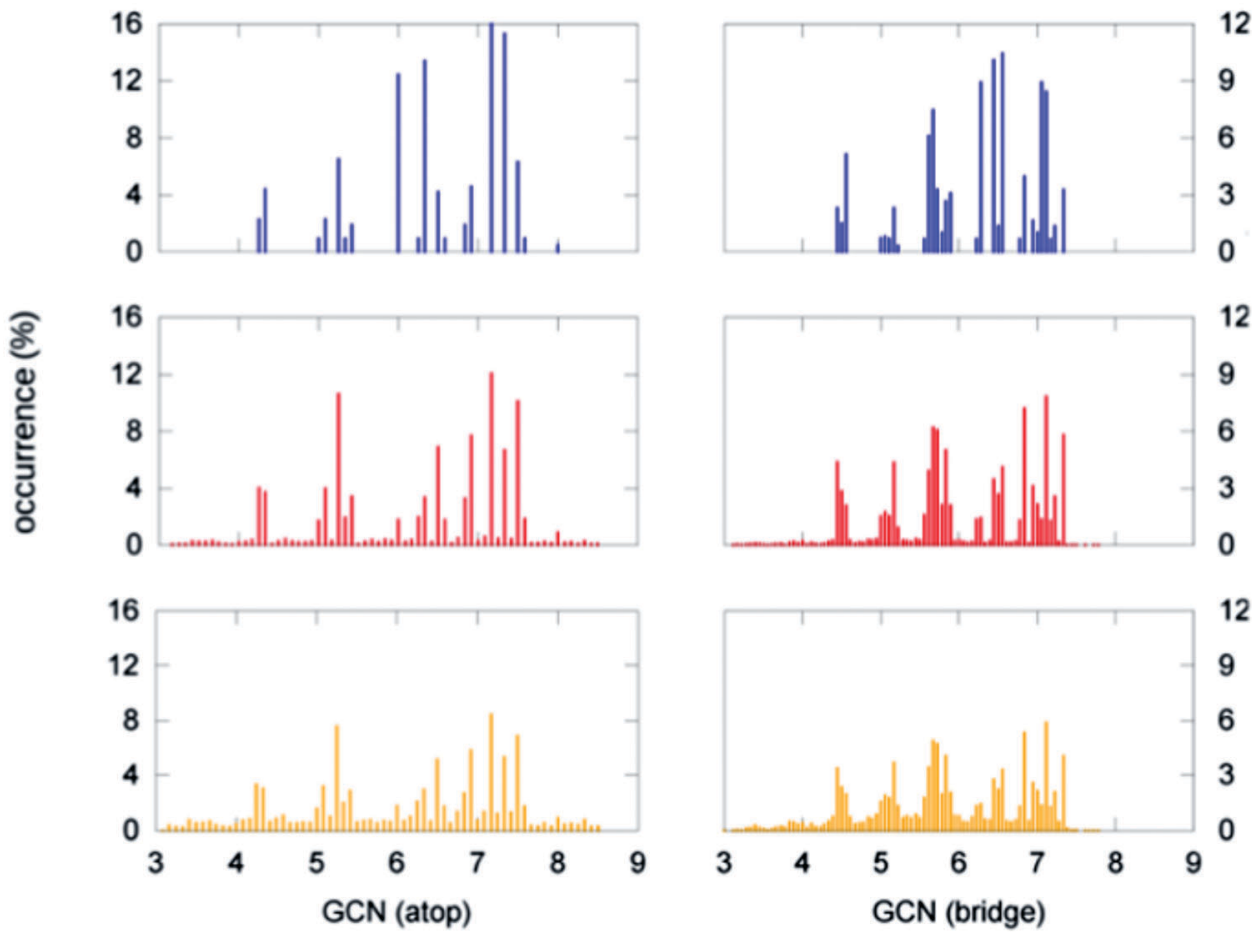

Fig. 8 A visual representation of the three populations discussed in the text in terms of their representative cluster distribution and shapes (A). The GCN genome of each population for atop and bridge adsorption modes (B). The more the sample loses its morphological identity, the broader its GCN genome becomes, presenting new values at both low and high GCNs, and decreasing the occurrence of its most abundant NEASs.

geometrical heterogeneity would produce a catalyst with a broader range of applications while a properly shape-selected nanocatalyst will be highly selective for a target reaction. We remark that the generalisation to any sample is straightforward. A sample is a collection of various isomers, each weighted accordingly to their probability of being observed. The choice of which structures should be included can be dictated by available experimental data, geometrical considerations, basin hopping, molecular dynamics simulations or a combination of them.

\subsection{From static to mobile: genomic evolution analysis}

The other feature of using the GCN genome is that we can monitor how dynamical structural rearrangements - including formation processes, e.g. growth, coalescence, and nucleation affect the catalytic properties. We refer to it as genomic evolution analysis, see Fig. 9, which aims to correlate catalytic properties to the structural changes, induced by a realistic environment. As the first application, we investigate the effect of heating and hence thermally activated processes, such as surface diffusion and island formation, on the GCN genome of two gas-phase monometallic Pt nanoparticles, namely the MDh of 585 and 1071 atoms. We report variations to their genome when subjected to a slow heating from $600 \mathrm{~K}$ to $1400 \mathrm{~K}$ by means of an iterative temperature molecular dynamics scheme, as implemented in the software LoDis. ${ }^{46-49}$ The interatomic potential is derived within the second moment approximation of the tight-binding, and the parameters are taken from ref. 50 and the heating rate is $25 \mathrm{~K} \mathrm{~ns}^{-1}$ where the temperature is controlled by an Andersen thermostat.

The evolution of the GCN genome for atop NEASs is given in Fig. 9 where their occurrence is highlighted by a different colour. Despite the absence of any major structural rearrangements 


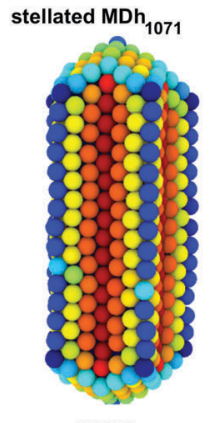

$600 \mathrm{~K}$

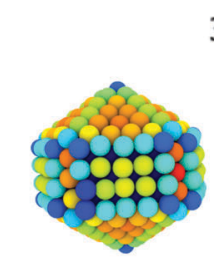

$600 \mathrm{~K}$

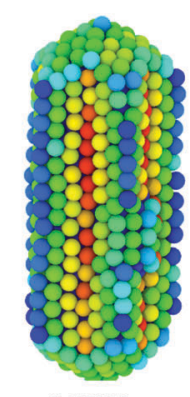

$1000 \mathrm{~K}$

3

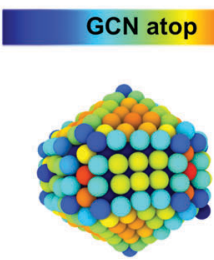

1000K

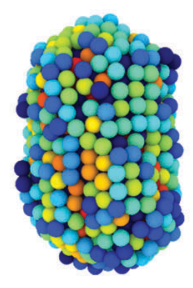

$1400 K$

9

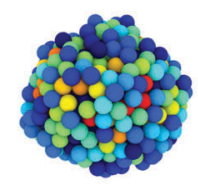

$1400 \mathrm{~K}$
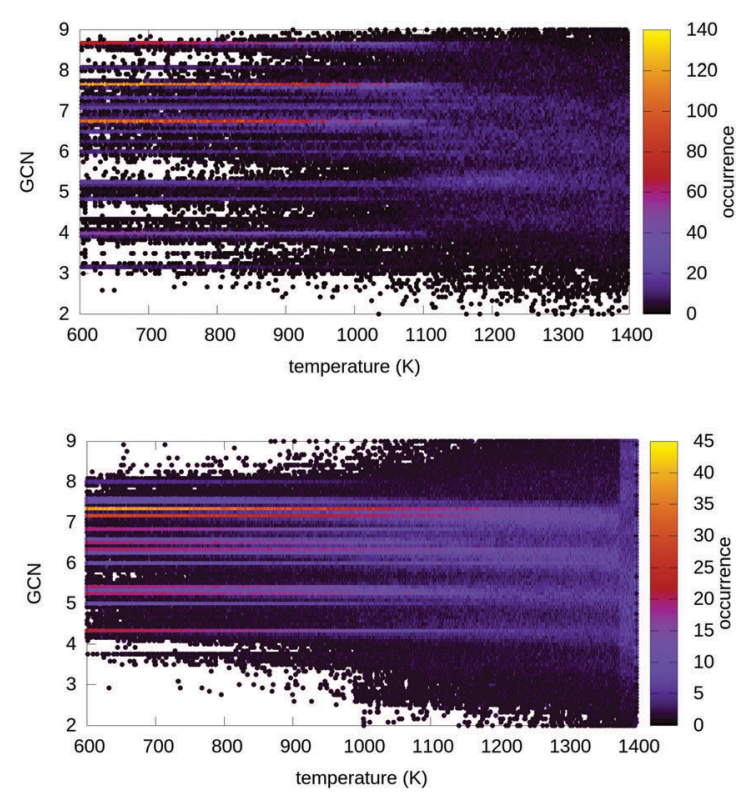

Fig. 9 Genome evolution analysis of elongated stellated $\mathrm{MDh}_{1071}(m=1, n=15, p=4)$ and $\mathrm{MDh}_{585}(m=5, n=4, p=2)$, snapshots taken at $600 \mathrm{~K}$, $1000 \mathrm{~K}$, and $1400 \mathrm{~K}$ where atop active sites are coloured according to their GCN (left) as a function of temperature. On the right, time/temperature evolution of the atop GCN genome for $\mathrm{MDh}_{1071}$ and $\mathrm{MDh}_{585}$, colour code refers to the occurrence of NEASs with a specific atop GCN value. The formation of defects is associated with the appearance of NEASs with GCNs different from the ones of the closed shell structure. Surface diffusion happening between 1000 and $1300 \mathrm{~K}$ is highlighted by a smoothing of the GCN genome. The melting transition at $1400 \mathrm{~K}$ corresponds to a continuum in the GCN fingerprint.

below $1350 \mathrm{~K}$, surface diffusion may yet take place. The melting transition onset is evident close to $1400 \mathrm{~K}$, where all sites become roughly equiprobable and the GCN genome loses its well-defined character, see Fig. 9. Before any phase change takes place, we recognise three temperature regimes: (i) the genome is roughly constant below $600 \mathrm{~K}$; (ii) activation of the surface diffusion of atoms lying on (111)/(111) edges, which leads to a first broadening of the GCN genome and the appearance of various sites characterised by a GCN $<5$ in the $600-1100 \mathrm{~K}$ range; (iii) above $1100 \mathrm{~K}$, we observe a continuous loss of the re-entrance features leading to an almost uniform population of the GCN values with a slightly higher occurrence of sites with a GCN between 4 and 7, although this occurrence might depend on the peculiar shape, the elongated $\mathrm{MDh}_{1071}$ has $10-15 \%$ of sites in the $1100-1300 \mathrm{~K}$ range, while the $\mathrm{MDh}_{585}$ spreading hits $20-40 \%$ up to $1300 \mathrm{~K}$.

\section{Tailored Pt nanoparticles for ORR}

To illustrate how the proposed approach can provide the rational design of a nanocatalyst, we focus on the oxygen reduction reaction catalysed by $\mathrm{Pt}$ nanoparticles. In agreement with the correlation between the weakening of the adsorption energy of $\mathrm{OH}$ (and atomic O) with respect to $\mathrm{Pt}(111)$, and the increase in the overall ORR activity of Pt catalysts, ${ }^{51}$ we have used the established linear dependence between the adsorption energy of $\mathrm{OH}$ and the atop-GCN to forecast the catalytic activity of each Pt-nanoparticle. We would like to highlight that the NEASs associated with atop GCN values between 7.5 and 8.3 are predicted to be the most active for ORR, where the smallest extreme of this range stands for atop sites on a perfect (111) surface. $^{25}$

The analysis of the size dependent trends in the geometrical genome allows us to easily predict a decrease in the mass activity of $\mathrm{Pt}$ nanoparticles below $2 \mathrm{~nm}$ in diameter in the catalysis of ORR. This behaviour can be easily inferred from the lack of active sites characterised by a GCN between 7.33 and 8.5, see Fig. 3. Furthermore, although the consensus is not universal, it is commonly found that the mass activity for cluster peaks is between 2.2 and $3 \mathrm{~nm} \cdot{ }^{52-54}$ This is the smallest size at which active sites with GCN in the ideal range appear. Similarly, increasing the size of the nanoparticle, the specific activity is known to gradually fade to that of the extended lowMiller index surfaces. This consideration can be inferred from the GCN genome of convex structures, where the number of active sites on low-Miller index facets grows more rapidly than the edge and vertex sites. For anisotropic and concave shapes with re-entrance edges of a similar length of the short axes, the ratio between high coordinated sites with GCN (atop) $>7.5$ and low-Miller index facets with a GCN(atop) $=7.5$ and GCN(atop) = 6.6, for (111) and (100) facets, respectively, is greater than 0.5, also at large sizes, leading to an enhancement of the activity with respect to low Miller index surfaces.

As concave sites are identified as ideal to efficiently catalyse ORR, we investigate the cuts of a pentagonal bi-pyramid, which maximally enhance their presence. The three cuts of a Dh can be identified by a different choice of three integers, $m, n$ and $p$, the number of atoms delimiting the (100) facets, and the depth 


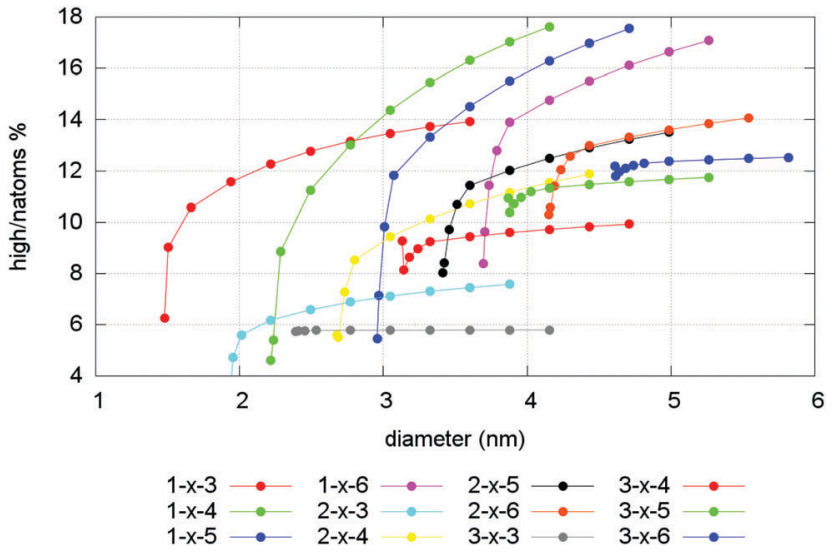

Fig. 10 Size-dependent density of adsorption sites with $7.5 \leq \mathrm{GCN} \leq$ 8.35 in Marks-decahedra, presenting $m$ in the 1-3 range, $p$ between 2 and 6 atoms, and $n$ in the 1-10 range. The latter is taken as the independent variable in the plot. The diameter (in $\mathrm{nm}$ ) refers to the maximum pair atomic distance.

of the re-entrances along the twin planes, respectively. Exploring systematically the $m, n, p$ parameter space, as sketched in Fig. 10, we observe that the relative occurrence of sites with GCN(atop) $>7.5$ with respect to the other surface sites and the total number of atoms is optimized for twinned shapes elongated along their five-fold axis, with a shorter diameter of $2 \mathrm{~nm}$ and a longer of one at least $3 \mathrm{~nm}$. Following the $m, n, p$ notation, it can be concluded that the best mNPs belong to the $1-n-4$ family, with $n>6$.

We would like to note that the synthesis of rationally designed nanostructures should balance site coordination and structural stability. Although an extensive study of the energetics and thermal stability of Pt-nanoparticles is out of the scope of this research paper, stellated twinned decahedra were chosen as representative of a wide category of concave structures observed in experiments. ${ }^{55,56}$ We would like to note that stellated nanostructures have been already demonstrated to be extremely active. ${ }^{57}$ In the first instance, their enhanced activity was attributed to the high density of vertex, edge, and generally low coordinated sites as well as a large surface area. In the light of the propose genome classification, we are prone to interpret those experimental results in terms of the presence of adsorption sites lying on re-entrances, which exhibit generalised coordination values corresponding to a high activity.

\section{Conclusions}

In summary, we propose an elegant and effective approach to deliver practical guidelines for tailoring metallic nanoparticles for target applications. Sequencing a genome based on a descriptor that bridges the electronic structure features to geometrical properties, we show that design rules can be extracted by high throughput screening simulations. Specifically, by the systematic analysis of the genome based on the generalised coordination number, we forecast truncated stellated twinned shapes, with pronounced re-entrances and very small, if not absent, (100) facets, elongated along their fivefold axis, as optimal catalysts for ORR. Moreover, the negative effects associated with vibrations and thermally activated processes, which determine a net broadening of the GCN genome of the gas-phase Pt-nanocatalysts, are negligible below $1000 \mathrm{~K}$. Changes in the activity should also be expected as a result of structural changes triggered by aggressive experimental environments due to leaching and surface roughening.

Our conclusions thrive from the fundamental studies that found a scaling relationship between catalyst ORR activity and the generalised coordination number, determined via accurate quantum-mechanical calculations. Our work calls for new and more accurate atomistic studies explicitly correlating activity to the catalyst architecture - morphology and size - in order to extend our approach to other chemical reactions. ${ }^{33}$ We also hope to inspire and motivate new experiments that can unequivocally determine the role of nanoarchitectures for improving ORR.

\section{Author contributions}

The work started from an original idea of FB. KR and GGA wrote the original code for the calculus of the generalized coordination number; $\mathrm{FB}$ and $\mathrm{KR}$ are the main developers of LoDiS and all the authors coded specific tools to generate geometrical structures. KR and GGA performed all the calculations. All the authors analysed the data and wrote the manuscript.

\section{Conflicts of interest}

There are no conflicts to declare.

\section{Acknowledgements}

GGA and FB thank the "Towards an Understanding of Catalysis on Nanoalloys”' (TOUCAN) EPSRC Critical Mass Grant (No. EP/ J010812/1) as does KR (Grant Reference ER/M506357/1). GGA is grateful to the postdoctoral scheme offered by the NMS faculty. All the authors are grateful to the financial support offered by the Royal Society (No. RG 120207) via our membership of the UK's HEC Materials Chemistry Consortium, which is funded by the EPSRC (EP/L000202). This work used the UK Materials and Molecular Modelling Hub for computational resources, MMM Hub, which is partially funded by the EPSRC (EP/P020194).

\section{References}

1 H. S. Taylor, Proc. R. Soc. A, 1925, 108, 105-111.

2 H. E. Grenga and K. R. Lawless, J. Appl. Phys., 1972, 43, 1508-1514.

3 L. E. Cratty and A. V. Granato, J. Chem. Phys., 1957, 26, 96-97.

4 G. G. Ertl, Handbook of heterogeneous catalysis., Wiley-VCH, 2008.

5 H. B. Schlegel, J. Comput. Chem., 2003, 24, 1514-1527. 
6 F. Abild-Pedersen, Catal. Today, 2016, 272, 6-13.

7 J. K. Nørskov, J. Rossmeisl, A. Logadottir, L. Lindqvist, J. R. Kitchin, T. Bligaard and H. Jónsson, J. Phys. Chem. B, 2004, 46, 17886-17892.

8 J. K. Nørskov, T. Bligaard, B. Hvolbæk, F. Abild-Pedersen, I. Chorkendorff and C. H. Christensen, Chem. Soc. Rev., 2008, 37, 2163.

9 B. Hammer and J. Nørskov, Adv. Catal., 2000, 45, 71-129.

10 X. Ma and H. Xin, Phys. Rev. Lett., 2017, 118, 036101.

11 C. R. Catlow, M. Davidson, C. Hardacre and G. J. Hutchings, Philos. Trans. R. Soc., A, 2016, 374, 20150089.

12 Y. Zhou, C. Jin, Y. Li and W. Shen, Nano Today, 2018, 20, 101-120.

13 R. E. Palmer, L. Cao and F. Yin, Rev. Sci. Instrum., 2016, 87, 046103.

14 Z. W. Wang and R. E. Palmer, Phys. Rev. Lett., 2012, 108, 245502.

15 R. Schlögl, Angew. Chem., Int. Ed., 2015, 54, 3465-3520.

16 I. Lee, F. Delbecq, R. Morales, M. A. Albiter and F. Zaera, Nat. Mater., 2009, 8, 132-138.

17 J. B. A. Davis, F. Baletto and R. L. Johnston, J. Phys. Chem. A, 2015, 119, 9703-9709.

18 D. M. Foster, R. Ferrando and R. E. Palmer, Nat. Commun., 2018, 9, 1323.

19 P. Strasser, Science, 2015, 349, 379-380.

20 C. Cui, L. Gan, M. Heggen, S. Rudi and P. Strasser, Nat. Mater., 2013, 12, 765-771.

21 A. Wilson, G. Kleen, D. Papageorgopoulos, R. Ahluwalia, B. James, C. Houchins and J. Huya-Kouadio, DOE Hydrogen and Fuel Cells Program Record, Fuel Cell System Cost, 2017.

22 F. Calle-Vallejo, J. I. Martínez, J. M. García-Lastra, P. Sautet and D. Loffreda, Angew. Chem., Int. Ed., 2014, 53, 8316-8319.

23 F. Calle-Vallejo, J. Tymoczko, V. Colic, Q. H. Vu, M. D. Pohl, K. Morgenstern, D. Loffreda, P. Sautet, W. Schuhmann and A. S. Bandarenka, Science, 2015, 350, 185-189.

24 F. Calle-Vallejo, D. Loffreda, M. T. M. Koper and P. Sautet, Nat. Chem., 2015, 7, 403-410.

25 F. Calle-Vallejo, M. D. Pohl, D. Reinisch, D. Loffreda, P. Sautet and A. S. Bandarenka, Chem. Sci., 2017, 8, 2283-2289.

26 G. G. Asara, L. O. Paz-Borbón and F. Baletto, ACS Catal., 2016, 6, 4388-4393.

27 L. Paz-Borbón, F. Baletto, L. O. Paz-Borbón and F. Baletto, Inorganics, 2017, 5, 43.

28 R. Kortlever, J. Shen, K. J. P. Schouten, F. Calle-Vallejo and M. T. M. Koper, J. Phys. Lett., 2015, 6, 4073-4082.

29 H. Huang, A. Ruditskiy, S.-I. Choi, L. Zhang, J. Liu, Z. Ye and Y. Xia, ACS Appl. Mater. Interfaces, 2017, 9, 31203-31212.

30 S. M. Alia, C. Ngo, S. Shulda, M.-A. Ha, A. A. Dameron, J. N. Weker, K. C. Neyerlin, S. S. Kocha, S. Pylypenko and B. S. Pivovar, ACS Omega, 2017, 2, 1408-1418.

31 T. Bian, H. Zhang, Y. Jiang, C. Jin, J. Wu, H. Yang and D. Yang, Nano Lett., 2015, 15, 7808-7815.
32 T. Nilsson Pingel, M. Jørgensen, A. B. Yankovich, H. Grönbeck and E. Olsson, Nat. Commun., 2018, 9, 2722.

33 Z. Zhao, Z. Chen, X. Zhang and G. Lu, J. Phys. Chem. C, 2016, 49, 28125-28130.

34 K. Rossi, PhD thesis, King's College London, 2018.

35 L. G. Verga, A. E. Russell and C.-K. Skylaris, Phys. Chem. Chem. Phys., 2018, 20, 25918-25930.

36 V. A. Rigo, C. R. Miranda and F. Baletto, arXiv.org, 2018, 1804.07631.

37 H. Wang and W. An, Catal.: Sci. Technol., 2017, 7, 596-606. 38 W. An and P. Liu, J. Phys. Chem. C, 2013, 117, 16144-16149.

39 F. Calle-Vallejo and A. Bandarenka, ChemSusChem, 2018, 11, 1824-1828.

40 M. J. Piotrowski, P. Piquini and J. L. F. Da Silva, Phys. Rev. B: Condens. Matter Mater. Phys., 2010, 81, 155446.

41 J. Higham and R. H. Henchman, J. Phys. Chem. C, 2016, 145, 084108.

42 G. Tritsaris, J. P. Greeley, J. Rossmeisl and J. K. Nørskov, Catal. Lett., 2011, 141, 909-913.

43 J. Aarons, L. Jones, A. Varambhia, K. E. MacArthur, D. Ozkaya, M. Sarwar, C.-K. Skylaris and P. D. Nellist, Nano Lett., 2017, 17, 4003-4012.

44 E. Ringe, R. P. Van Duyne and L. D. Marks, J. Phys. Chem. C, 2013, 117, 15859-15870.

45 G. Rossi and R. Ferrando, J. Phys.: Condens. Matter, 2009, 21, 084208.

46 F. Baletto et al., LoDiS: Low Dimensional System Molecular Dynamics, 2018, http://balettogroup.weebly.com.

47 K. Rossi, L. Pavan, Y. Soon and F. Baletto, Eur. Phys. J. B, 2018, 91, 33.

48 K. Rossi, T. Ellaby, L. O. Paz-Borbón, I. Atanasov, L. Pavan and F. Baletto, J. Phys.: Condens. Matter, 2017, 29, 145402.

49 F. Baletto, C. Mottet and R. Ferrando, Chem. Phys. Lett., 2002, 354, 82-87.

50 F. Baletto, R. Ferrando, A. Fortunelli, F. Montalenti and C. Mottet, J. Chem. Phys., 2002, 116, 3856-3863.

51 J. K. Nørskov, J. Rossmeisl, J. Logadottir, A. Lindqvist, L. Kitchin, T. J. R. Bligaard and H. Jonsson, J. Phys. Chem. $B, 2004,108,17886-17892$.

52 M. Shao, A. Peles and K. Shoemaker, Nano Lett., 2011, 11, 3714-3719.

53 L. Li, F. Abild-Pedersen, J. Greeley and J. K. Nørskov, J. Phys. Chem. Lett., 2015, 6, 19.

54 J. Kleis, J. Greeley, N. A. Romero, V. A. Morozov, H. Falsig, A. H. Larsen, J. Lu, J. J. Mortensen, M. Dułak, K. S. Thygesen, J. K. Nørskov and K. W. Jacobsen, Catal. Lett., 2011, 141, 1067-1071.

55 Y. Feng, F. Ye, H. Liu and J. Yang, Sci. Rep., 2015, 5, 16219. 56 H. Liu, F. Ye, Q. Yao, H. Cao, J. Xie, J.-Y. Lee and J. Yang, Sci. Rep., 2014, 4, 3969.

57 H. Liu, F. Ye, Q. Yao, H. Cao, J. Xie, J. Y. Lee and J. Yang, Sci. Rep., 2015, 4, 3969. 